\title{
Sex-associated genome region of poplar
}

Pushkova E.N.*, Beniaminov A.D., Borkhert E.V., Melnikova N.V., Dmitriev A.A.

Engelhardt Institute of Molecular Biology, RAS, Moscow, Russia

*e-mail:pushkova18@gmail.com

Poplars (genus Populus) are dioecious plants, and sexual dimorphism in males and females is actively studied. Recent investigations on the determination of poplar sex revealed sex-associated single nucleotide polymorphisms (SNPs) and genome regions of their localization. However, the improvement of the genome assembly is necessary for accurate mapping of the sex locus. The key genes that are involved in poplar sex determination are still unknown. The goal of our work was the obtaining of high-quality genome assembly of Populus $\times$ sibirica, which is the most common poplar in Moscow, and identification of sex-specific DNA polymorphism. Twenty male and twenty female plants of $P$. $\times$ sibirica were collected in different regions of the Moscow city. DNA was extracted and amplicon libraries for sequencing on the Illumina platform were prepared. Deep sequencing of genes encoding MET1 and homolog of ARR17, which contain a number of sex-associated SNPs, was performed for the 40 samples on MiSeq (Illumina), and about 4000x coverage was obtained for each sample. We identified 17 (11 in MET1 and 6 in ARR17) sex-specific SNPs. All females were homozygous, while all males were heterozygous for identified sex-specific SNP sites. Moreover, in one allelic variant, males had the same nucleotides as females, while in the other one - sex-specific SNPs. Thus, the colocation of sex-specific SNPs was revealed. Two males and two females of $P . \times$ sibirica were selected for the whole genome sequencing. The Nanopore platform with long reads and the Illumina one with high-precision short reads were used. Combination of these sequencing platforms enabled high-quality genome assembly and accurate identification of the sex-specific locus in $P . \times$ sibirica genome. Obtained results contribute to the understanding of the mechanisms of poplar sex determination.

Acknowledgements: This work was funded by the RFBR, grant 18-34-20113 mol_a_ved. 